\title{
Existence and Uniqueness for Stochastic Dynamic Equations
}

\author{
David Grow $^{1} \&$ Suman Sanyal ${ }^{2}$ \\ ${ }^{1}$ Department of Mathematics and Statistics, Missouri University of Science and Technology, Rolla, USA \\ ${ }^{2}$ Department of Mathematics, Marshall University, Huntington, USA \\ Correspondence: Suman Sanyal, Department of Mathematics, Marshall University, Huntington, WV 25755, USA. \\ Tel: 1-304-696-3049. E-mail: sanyal@ marshall.edu
}

Received: March 10, 2013 Accepted: April 8, 2013 Online Published: April 16, 2013

doi:10.5539/ijsp.v2n2p77 URL: http://dx.doi.org/10.5539/ijsp.v2n2p77

\begin{abstract}
The theory of stochastic dynamic equations extends and unifies the theories of stochastic difference and differential equations. In this paper, we prove the existence and uniqueness of the strong solution of a certain class of stochastic dynamic equations. As a principal tool in the proof, we define and develop the properties of stochastic dynamic integrals with respect to a Brownian motion indexed by a time scale. Finally we illustrate our theory with the examples of stochastic exponential and geometric Brownian motion.
\end{abstract}

Keywords: stochastic dynamic equations, stochastic dynamic integral, Brownian motion, time scale

\section{Introduction}

The theory of stochastic dynamic equations ( $\mathrm{S} \Delta \mathrm{E})$ is a relatively new area of mathematics that extends and unifies the theory of stochastic difference and differential equations. The basic idea is to replace the time domain of the underlying stochastic process with a time scale, $\mathbb{T}$; that is $\mathbb{T}$ is an arbitrary nonempty closed subset of the reals $\mathbb{R}$. It is evident that when $\mathbb{T}=\mathbb{Z}$ we have the theory of stochastic difference equations and when $\mathbb{T}=\mathbb{R}$ we have the theory of stochastic differential equations. The theory of $\mathrm{S} \Delta \mathrm{E}$ was first investigated in (Sanyal, 2008). Subsequently various authors have contributed to this endeavor. Notable contributions include the construction of Brownian motion in (Grow \& Sanyal, 2011), the quadratic variation of Brownian motion in (Grow \& Sanyal, 2012), the construction of the stochastic dynamic exponential and the explicit solution of geometric Brownian motion in (Bohner \& Sanyal, 2010), the existence and uniqueness of random dynamical systems in (Lugan \& Lupulescu, 2012), and in the special case of $q$-calculus time scale $\mathbb{T}=q^{\mathbb{Z}^{+}}=\left\{1, q, q^{2}, q^{3}, \ldots\right\}$ for $q>1$, Itô's lemma and a financial option pricing application in (Haven, 2009, 2011).

In this paper we provide conditions which guarantee the existence and uniqueness of the strong solution of a certain class of stochastic dynamic initial value problems (IVPs) of the form

$$
\Delta X=a(X, t) \Delta t+b(X, t) \Delta W, \quad X_{t_{0}}=X_{0} .
$$

By a strong solution of $(1)$ on $(\Omega, \mathcal{F}, \mathbb{P})$ and w.r.t. a fixed Brownian motion $W$ and a fixed initial process $X_{0}$, we mean a stochastic process indexed by a time scale, $X=\left\{X_{s}: s \in \mathbb{T}\right\}$, with sample paths that are continuous and with the following properties:

1) $X$ is adapted to the filtration $\left\{\mathcal{F}_{s}: s \in \mathbb{T}\right\}$,

2) $\mathbb{P}\left(X_{t_{0}}=X_{0}\right)=1$,

3) $\mathbb{P}\left(\int_{\mathbb{T}_{t}}\left(\left|a\left(X_{u}, u\right)\right|+b^{2}\left(X_{u}, u\right)\right) \Delta u<\infty\right)=1$,

and the integral version of (1)

$$
X_{t}=X_{0}+\int_{\mathbb{T}_{t}} a\left(X_{u}, u\right) \Delta u+\int_{\mathbb{T}_{t}} b\left(X_{u}, u\right) \Delta W_{u},
$$

holds almost surely. We define Brownian motion indexed by $\mathbb{T}$ as an adapted, continuous stochastic process $W=\left\{W_{t}, \mathcal{F}_{t}: t \in \mathbb{T}\right\}$, defined on $(\Omega, \mathcal{F}, \mathbb{P})$ such that 
1) $W_{t_{0}}=0$ a.e.- $\mathbb{P}$;

2) if $t_{0} \leq s<t$ and $s, t \in \mathbb{T}$ then the increment $W_{t}-W_{s}$ is normally distributed with mean 0 and variance $t-s$ and is independent of $\mathcal{F}_{s}$.

For a generalization of the Kolmogorov-Čentsov theorem and the construction of such a generalized Brownian motion indexed by $\mathbb{T}$, please refer to (Grow \& Sanyal, 2011). Here and in the rest of this paper $\mathbb{T}_{t}$ denotes $\left[t_{0}, t\right] \cap \mathbb{T}$ for some $t \in \mathbb{T}$. In (2), the first integral is a $\Delta$-integral defined in (Bohner \& Peterson, 2001) and the validation for the second integral is provided in Section 3.

The paper is organized as follows. In Section 2 we provide basic definitions regarding time scales, in Section 3 we define the stochastic integral w.r.t. a Brownian motion indexed by a time scale and develop some its properties, and finally in Section 4 we state and prove an existence and uniqueness result concerning (1).

\section{Preliminaries}

A time scale, $\mathbb{T}$, is primarily used to unify and extend discrete and continuous analysis (Bohner \& Peterson, 2001, 2003). Examples of time scales include $\mathbb{R}, \mathbb{Z}, q^{\mathbb{N} 0}$ for $q>1, h \mathbb{Z}$ for $h>0,[0,1] \cup[2,3]$, the Cantor set, etc. Given a time scale, $\mathbb{T}$, we define $\rho(t)=\sup \{s \in \mathbb{T}: s<t\}$ as the backward shift operator $\rho$ on $\mathbb{T}^{k}, \sigma(t)=\inf \{s \in \mathbb{T}$ : $s>t\}$ as the forward shift operator $\sigma$ on $\mathbb{T}_{k}$, and $\mu(t)=\sigma(t)-t$ as the graininess function depicting the gaps between two points in a time scale, where the sets $\mathbb{T}^{k}$ and $\mathbb{T}_{k}$ are defined as $\mathbb{T}^{k}:=\mathbb{T} \backslash\{\sup \mathbb{T}\}$ and $\mathbb{T}_{k}:=\mathbb{T} \backslash\{\inf \mathbb{T}\}$. For the definitions of (i) right dense point, (ii) the set of right dense continuous functions $\left(C_{\mathrm{rd}}\right)$, (iii) the set of all regressive functions $(\mathcal{R})$, (iv) the set of positively regressive functions $\left(\mathcal{R}^{+}\right)$, (v) Hilger derivative (or $\Delta$-derivative), (vi) $\Delta$-anti-derivative, and (vi) $\Delta$-integral, we refer the readers to (Bohner \& Peterson, 2001). For $t_{0} \in \mathbb{T}, y_{0} \in \mathbb{R}$, and $p \in \mathcal{R}$, the IVP

$$
y^{\Delta}=p(t) y, \quad y\left(t_{0}\right)=y_{0}
$$

has a unique solution given by $y=e_{p}\left(\cdot, t_{0}\right) y_{0}$. The generalized polynomials $g_{k}, h_{k}: \mathbb{T} \times \mathbb{T} \rightarrow \mathbb{R}, k \in \mathbb{N}_{0}$ are defined as follows. The functions $g_{0}$ and $h_{0}$ are $g_{0}(t, s)=h_{0}(t, s) \equiv 1$ for all $s, t \in \mathbb{T}$, and for $k \in \mathbb{N}_{0}$

$$
h_{k+1}^{\Delta}(t, s)=h_{k}(t, s)
$$

for $t \in \mathbb{T}^{K}$ with $h_{k+1}(s, s)=0$ where $h_{k}^{\Delta}(t, s)$ denotes for each fixed $s$ the $\Delta$-derivative of $h_{k}(t, s)$ w.r.t. $t$. Similarly $g_{k+1}^{\Delta}(t, s)=g_{k}(\sigma(t), s)$ for $t \in \mathbb{T}_{k}$ with $g_{k+1}(s, s)=0$. Thence, it can be shown that

$$
e_{k}\left(t, t_{0}\right)=\sum_{n=0}^{\infty} k^{n} h_{n}\left(t, t_{0}\right)
$$

for constant $k \in \mathcal{R}$ and $t_{0}, t \in \mathbb{T}$. To verify (4) we need the following lemma.

Lemma 1 Let $s, t \in \mathbb{T}$ with $s \leq t$ and $h$ defined as in (3). Then

$$
h_{n}(t, s) \leq \frac{(t-s)^{n}}{n !}
$$

for $n \in \mathbb{N}_{0}$.

Proof. Observe that $h_{0}(t, s)=1$ and $h_{1}(t, s)=t-s$ so (5) holds when $n=0$ or 1 . Suppose (5) holds for some integer $n \geq 1$. Then

$$
h_{n+1}(t, s)=\int_{s}^{t} h_{n}(u, s) \Delta u \leq \frac{1}{n !} \int_{s}^{t}(u-s)^{n} \Delta u .
$$

But

$$
\left((t-s)^{n+1}\right)^{\Delta}=\sum_{v=0}^{n}(\sigma(t)-s)^{v}(t-s)^{n-v}
$$

and

$$
(u-s)^{n} \leq \sum_{v=0}^{n} \frac{1}{n+1}(\sigma(u)-s)^{v}(u-s)^{n-v}
$$


for $u \geq s$, so

$$
\begin{aligned}
\int_{s}^{t}(u-s)^{n} \Delta u & \leq \int_{s}^{t}\left(\frac{1}{n+1} \sum_{v=0}^{n}(\sigma(u)-s)^{v}(u-s)^{n-v}\right) \Delta u \\
& =\int_{s}^{t}\left(\frac{1}{n+1}(u-s)^{n+1}\right)^{\Delta} \Delta u \\
& =\frac{(t-s)^{n+1}}{n+1} .
\end{aligned}
$$

Substituting in (6) gives

$$
h_{n+1}(t, s) \leq \frac{1}{n !} \frac{(t-s)^{n+1}}{(n+1) !}=\frac{(t-s)^{n+1}}{(n+1) !} .
$$

Hence (5) holds for all $n \in \mathbb{N}_{0}$ by induction.

To begin the verification of (4), let $y_{N}(t)=\sum_{n=0}^{N} k^{n} h_{n}\left(t, t_{0}\right)$ for $N \in \mathbb{N}_{0}$. Then

$$
y_{N}^{\Delta}(t)=\sum_{n=0}^{N} k^{n} h_{n}^{\Delta}\left(t, t_{0}\right)=\sum_{n=1}^{N} k^{n} h_{n-1}^{\Delta}\left(t, t_{0}\right)=k \sum_{n=0}^{N-1} k^{n} h_{n}^{\Delta}\left(t, t_{0}\right)=k y_{N-1}(t) .
$$

From Lemma 1, it follows that

$$
\sum_{n=0}^{\infty}\left|k^{n} h_{n}\left(t, t_{0}\right)\right| \leq \sum_{n=0}^{\infty} \frac{k^{n}\left(t-t_{0}\right)^{n}}{n !}=e^{k\left(t-t_{0}\right)}<\infty
$$

Therefore $\left\{y_{N}^{\Delta}\right\}_{N=1}^{\infty}=\left\{k y_{N-1}\right\}_{N=1}^{\infty}$ converges uniformly on $\mathbb{T}_{T}$ and the limit function

$$
y(t)=\lim _{N \rightarrow \infty} y_{N}(t)=\sum_{n=0}^{\infty} k^{n} h_{n}\left(t, t_{0}\right)
$$

is differentiable on $\mathbb{T}_{T}$ with

$$
y^{\Delta}(t)=\lim _{N \rightarrow \infty} y_{N}^{\Delta}(t)=k \lim _{N \rightarrow \infty} y_{N-1}(t)=k y(t)
$$

Clearly $y\left(t_{0}\right)=\sum_{n=0}^{\infty} k^{n} h_{n}\left(t, t_{0}\right)=1$, so $y(t)=\sum_{n=0}^{\infty} k^{n} h_{n}\left(t, t_{0}\right)$ is a solution to the IVP $y^{\Delta}=k y, y\left(t_{0}\right)=1$ on $\mathbb{T}$. By uniqueness of solutions to this IVP, $y(t)=e_{k}\left(t, t_{0}\right)$ on $\mathbb{T}$, i.e. (4) holds for all $t \in \mathbb{T}$. Finally we present the following lemma (an analogue of Gronwall's inequality) without proof.

Lemma 2 Let $\phi \in \mathrm{C}_{\mathrm{rd}}, f \in \mathcal{R}^{+}, f \geq 0$, and let $C_{0} \in \mathbb{R}$. Then

$$
\phi(t)=C_{0}+\int_{\mathbb{T}_{t}} f(u) \phi(u) \Delta u
$$

for all $t \in \mathbb{T}_{T}$ implies

$$
\phi(t) \leq C_{0} e_{f}\left(t, t_{0}\right)
$$

for all $t \in \mathbb{T}_{T}$.

In the next section we present the construction of the stochastic dynamic integral.

\section{Stochastic Dynamic Integral}

Let inf $\mathbb{T}>-\infty$ and $\sup \mathbb{T}=\infty$ and let $\psi$ be a real, increasing, and right continuous function on $\mathbb{T}$. LebesgueStieltjes delta integrals w.r.t. $\psi$ on intervals in $\mathbb{T}$ can be defined in terms of Lebesgue-Stieljes intervals on intervals in $\mathbb{R}$ in a manner analogous to the Lebesgue delta integral w.r.t. the forward shift function of $\mathbb{T}$ (Bohner \& Peterson, 2003; Guseinov, 2003). Explicitly, define $\Psi$ on $\mathbb{R}$ by $\Psi(t)=\inf \{\psi(s): s \in \mathbb{T}, t<s\}$. Since $\Psi$ is real, increasing, and right continuous of $\mathbb{R}$ such that $v(c, d]=\Psi(d)-\Psi(c)$ for all intervals in $\mathbb{R}$ of the form $(c, d]$. If $\phi$ is a Borel 
measurable function on an interval $I$ such that $\int_{I}|\phi(x)| \mathrm{d} v<\infty$ then we say that $\phi$ is Lebesgue-Stieltjes integrable w.r.t. $\Psi$ on $I$.

Definition 1 Let $a<b$ be points in $\mathbb{T}$, let $\psi$ be a real, increasing, and right continuous function on $[a, b] \cap \mathbb{T}$, and let $\phi$ be a Borel measurable function which is Lebesgue-Stieltjes integrable w.r.t. $\Psi$ on $[a, b]$. Then the LebesgueStieltjes delta integral of $\phi$ w.r.t. $\psi$ on the interval $[a, b] \cap \mathbb{T}$ is defined by

$$
\int_{a}^{b} \phi(x) \Delta \psi(x)=\int_{[a, b]} \phi(x) \mathrm{d} \Psi(x)=\int_{[a, b]} \psi^{+} \mathrm{d} v-\int_{[a, b]} \psi^{-} \mathrm{d} v .
$$

Similarly, if $\phi$ is a nonnegative Borel measurable function on $[a, b]$, then we define

$$
\int_{a}^{b} \phi(x) \Delta \psi(x)=\int_{[a, b]} \phi(x) \mathrm{d} \Psi(x)=\int_{[a, b]} \psi \mathrm{d} v,
$$

with $+\infty$ as a possible value of this integral.

For the construction of the stochastic dynamic integral $\int_{\mathbb{T}_{t}} X_{u} \Delta W_{u}$, we parallel the construction of the stochastic integral w.r.t. a continuous square integrable martingale on $[0, \infty)$ as presented in (Karatzas \& Shreve, 1998). Let $W=\left\{W_{t}, \mathcal{F}_{t}: t \in \mathbb{T}\right\}$ be a Brownian motion indexed by $\mathbb{T}$ defined on $(\Omega, \mathcal{F}, \mathbb{P})$. In order to avoid possible measure theoretic pathologies, we can and do assume that $\mathcal{F}_{t_{0}}$ contains all subsets of $\mathbb{P}$-measure zero in $\mathcal{F}$ and the filtration $\left\{\mathcal{F}_{t}: t \in \mathbb{T}\right\}$ is right continuous, i.e.

$$
\mathcal{F}_{t}=\cap\left\{\mathcal{F}_{\rho(s)}: s \in \mathbb{T}, s>t\right\}
$$

for all $t \in \mathbb{T}$. By a partition of the interval $\mathbb{T}_{t}$ we mean a finite subset $\mathcal{P}: t_{0}<t_{1}<t_{2}<\cdots<t_{n}=t$ of $\mathbb{T}_{t}$ and we define $\operatorname{mesh}(\mathcal{P}):=\max _{1 \leq i \leq n}\left|t_{i}-t_{i-1}\right|$ for which $\left(t_{i-1}, t_{i}\right) \cap \mathbb{T} \neq \emptyset$ and we discard intervals for which $\left(t_{i-1}, t_{i}\right) \cap \mathbb{T}=\emptyset$.

Definition $2 X$ be a stochastic process indexed by $\mathbb{T}$ with inf $\mathbb{T}>-\infty$. Then the quadratic variation of $X$ over the partition $\mathcal{P}$ of the time scale $\mathbb{T}_{t}$ is defined as

$$
X_{t_{0}, t}^{2}(\mathcal{P})=\sum_{k=1}^{n}\left(X_{t_{k}}-X_{t_{k-1}}\right)^{2} .
$$

If $X_{t_{0}, t}^{2}(\mathcal{P})$ converges in probability sufficiently rapidly as $\operatorname{mesh}(\mathcal{P}) \rightarrow 0$ then the limit, $\langle X\rangle_{t}$, is called the quadratic variation of $X$ on $\mathbb{T}_{t}$.

In (Grow \& Sanyal, 2012), it has been shown that

$$
\langle W\rangle_{t}=\lambda\left(\mathbb{T}_{t}\right)+\sum_{b_{n} \leq t}\left(W_{b_{n}}-W_{a_{n}}\right)^{2}
$$

where $\lambda$ denotes the Lebesgue measure and $\dot{\cup}_{n=1}^{\infty}\left(a_{n}, b_{n}\right)=\left[t_{0}, \infty\right) \backslash \mathbb{T}$.

For fixed $t \in \mathbb{T}$, the function $\omega \rightarrow\langle W\rangle_{t}(\omega)$ is in $L^{2}(\Omega, \mathbb{P})$ and so

$$
\sum_{b_{n} \leq t}\left(W_{b_{n}}-W_{a_{n}}\right)^{2}<\infty \quad \mathbb{P} \text {-a.e. } \omega \in \Omega \text {. }
$$

Then $\mathbb{P}$-a.e. $\omega \in \Omega$, the function $\omega \rightarrow\langle W\rangle_{t}(\omega)$ is real, increasing, and right continuous on $\mathbb{T}$. Consequently, we may define a measure $\mu_{W}$ on $(\mathbb{T} \times \Omega, \mathcal{B}(\mathbb{T}) \otimes \mathcal{F})$ by

$$
\mu_{W}(A)=\mathbb{E}\left(\int_{T} \chi_{A}(u, \cdot) \Delta\langle W\rangle_{u}\right)=\int_{\Omega} \int_{T} \chi_{A}(u, \omega) \Delta\langle W\rangle_{u}(\omega) \mathrm{d} \mathbb{P}(\omega) .
$$

We will say that two measurable, adapted processes $X=\left\{X_{s}, \mathcal{F}_{s}: s \in \mathbb{T}\right\}$ and $Y=\left\{Y_{s}, \mathcal{F}_{s}: s \in \mathbb{T}\right\}$ indexed by $\mathbb{T}$ are equivalent if $X_{s}(\omega)=Y_{s}(\omega)$ for $\mu_{W}$-a.e. $(s, \omega)$. Then

$$
[X]_{T}=\left(\int_{\Omega} \int_{\mathbb{T}_{T}} X_{u}^{2}(\omega) \Delta\langle W\rangle_{u}(\omega) \mathrm{d} \mathbb{P}(\omega)\right)^{1 / 2}
$$


for $t \in \mathbb{T}$ defines an $L^{2}$-norm for $X$ regarded as a function of $(s, \omega)$ restricted to the space $\mathbb{T}_{T} \times \Omega$ under the measure $\mu_{W}$, and $[X-Y]_{T}=0$ for all $T \in \mathbb{T}$ if and only if $X$ and $Y$ are equivalent.

Definition 3 A stochastic process $X=\left\{X_{t}: t \in \mathbb{T}\right\}$ on $(\Omega, \mathcal{F})$ is called progressively measurable w.r.t. the filtration $\left\{\mathcal{F}_{t}: t \in \mathbb{T}\right\}$ if the mapping $(s, \omega) \mapsto X_{s}(\omega)$ from $\left(\mathbb{T}_{t} \times \Omega, \mathcal{B}\left(\mathbb{T}_{t}\right) \otimes \mathcal{F}_{t}\right)$ into $(\mathbb{R}, \mathcal{B}(\mathbb{R}))$ is measurable for all $t \in \mathbb{T}$.

Let $\mathcal{L}^{*}$ denote the set of equivalent classes of stochastic processes $X$ which are progressively measurable w.r.t. $\left\{\mathcal{F}_{t}\right.$ : $t \in \mathbb{T}\}$ and for which $[X]_{T}<\infty$ for all $T \in \mathbb{T}$. Following the standard convention in measure and integration, we will not distinguish between an equivalence class in $\mathcal{L}^{*}$ and a representative process $X$ from that class. Choose and fix a strictly increasing sequence $\left\{t_{n}\right\}_{n=1}^{\infty}$ of points from $\mathbb{T}$ tending to infinity and define a metric on $\mathcal{L}^{*}$ by

$$
d(X, Y)=[X-Y] \quad \text { where } \quad[X]=\sum_{n=1}^{\infty} 2^{-n} \min \left\{1,[X]_{t_{n}}\right\} .
$$

Let $\mathcal{L}_{T}^{*}$ denote the set of processes $X$ in $\mathcal{L}^{*}$ for which $X_{t}(\omega)=0$ for all $t \in[T, \infty) \cap \mathbb{T}$ and $\omega \in \Omega$. Define $\mathcal{L}_{\infty}^{*}$ as the class of processes $X \in \mathcal{L}^{*}$ for which

$$
\mathbb{E}\left(\int_{\mathbb{T}} X_{u}^{2} \Delta\langle W\rangle_{u}\right)=\int_{\Omega} \int_{\mathbb{T}} X_{u}^{2}(\omega) \Delta\langle W\rangle_{u}(\omega) \mathrm{d} \mathbb{P}(\omega)<\infty .
$$

If $T \in \mathbb{T}$ or $T=\infty$ then $\mathcal{L}_{T}^{*}$ is a subspace of the Hilbert space $\mathcal{H}_{T}=L^{2}\left(\mathbb{T}_{T} \times \Omega, \mathcal{B}\left(\mathbb{T}_{t}\right) \otimes \mathcal{F}_{T}, \mu_{W}\right)$.

Definition 4 A process $X$ indexed by $\mathbb{T}$ is called simple if there exists a strictly increasing sequence $\left\{s_{n}\right\}_{n=0}^{\infty}$ of points from $\mathbb{T}$, tending to infinity with $s_{0}=t_{0}$, as well as a sequence of random variables $\left\{\xi_{n}\right\}_{n=0}^{\infty}$ and a constant $C<\infty$ with $\sup \left|\xi_{n}(\omega)\right| \leq C$ for every $\omega \in \Omega$, such that $\xi_{n}$ is $\mathcal{F}_{s_{n}}$-measurable for every $n \geq 0$ and

$$
X_{t}(\omega)=\sum_{i=0}^{\infty} \xi_{i}(\omega) \chi_{\left[s_{i-1}, s_{i}\right) \cap \mathbb{T}}(t)
$$

for all $t \in \mathbb{T}$ and $\omega \in \Omega$.

Denote by $\mathcal{L}_{0}$ the class of all simple processes and observe that $\mathcal{L}_{0} \subseteq \mathcal{L}^{*}$ since the processes in $\mathcal{L}_{0}$ are progressively measurable and bounded. A standard construction shows that the set $\mathcal{L}_{0}$ of simple processes is dense in $\mathcal{L}^{*}$ w.r.t. metric $d(X, Y)=[X-Y]$ defined in (9).

Definition 5 The Brownian motion transform of (10) in $\mathcal{L}_{0}$ is a progressively measurable stochastic process

$$
I(X)=\left\{I_{t}(X), \mathcal{F}_{t}: t \in \mathbb{T}\right\}
$$

given by

$$
I_{t}(X)=\sum_{i=0}^{n-1} \xi_{i}\left(W_{s_{i+1}}-W_{s_{i}}\right)+\xi_{n}\left(W_{t}-W_{s_{n}}\right) .
$$

Here $n \geq 0$ is the unique integer for which $s_{n} \leq t<s_{n+1}$.

It is apparent from Definition 5 that the Brownian motion transform is linear, i.e.

$$
I(\alpha X+\beta Y)=\alpha I(X)+\beta I(Y)
$$

for all $\alpha, \beta \in \mathbb{R}$ and all $X, Y \in \mathcal{L}_{0}$. Furthermore, if $X \in \mathcal{L}_{0}$ then $I(X)$ is a right continuous, square integrable martingale indexed by $\mathbb{T}$ with quadratic variation

$$
\langle I(X)\rangle_{t}=\int_{\mathbb{T}_{t}} X_{u}^{2} \Delta\langle W\rangle_{u}
$$

and second moment

$$
\mathbb{E}\left(I_{t}^{2}(X)\right)=\mathbb{E}\left(\int_{\mathbb{T}_{t}} X_{u}^{2} \Delta\langle W\rangle_{u}\right)=\int_{\mathbb{T}_{t}} \mathbb{E}\left(X_{u}^{2}\right) \Delta u .
$$

for all $t \in \mathbb{T}$. 
Definition 6 The space $\mathcal{M}_{2}$ of all right continuous, square integrable martingales indexed by $\mathbb{T}$ consists of those adapted processes $X=\left\{X_{t}, \mathcal{F}_{t}: t \in \mathbb{T}\right\}$ such that

1) $X_{t_{0}}=0$ a.e.-P;

2) for $\mathbb{P}$-a.e $\omega \in \Omega$, the function $t \mapsto X_{t}(\omega)$ is right continuous on $\mathbb{T}$;

3) $\mathbb{E}\left(X_{t}^{2}\right)=\int_{\Omega} X_{t}^{2}(\omega) \mathrm{d} \mathbb{P}(\omega)<\infty$ for all $t \in \mathbb{T}$;

4) the conditional expectations of $X$ satisfy $\mathbb{E}\left(X_{t} \mid \mathcal{F}_{s}\right)=X_{s}$ for all $s$ and $t$ in $\mathbb{T}$ such that $t_{0} \leq s \leq t$.

For any $X \in \mathcal{M}_{2}$, if we define

$$
\|X\|:=\sum_{n=1}^{\infty} 2^{-n} \min \left\{1, \sqrt{\mathbb{E}\left(X_{t_{n}}^{2}\right)}\right\},
$$

then $\|X-Y\|$ is a pseudo-metric on $\mathcal{M}_{2}$, almost all of whose sample paths $t \mapsto X_{t}(\omega)$ and $t \mapsto Y_{t}(\omega)$ are identical on $\mathbb{T}$, then $\|X-Y\|$ is a complete metric on the equivalence classes of $\mathcal{M}_{2}$. As usual, we will blur the distinction between an equivalence class in $\mathcal{M}_{2}$ and a representative process $X$ from that class. Observe that for $X \in \mathcal{L}_{0}$ we have

$$
\begin{aligned}
\|I(X)\| & =\sum_{n=1}^{\infty} 2^{-n} \min \left\{1, \sqrt{\mathbb{E}\left(I_{t_{n}}^{2}(X)\right)}\right\} \\
& =\sum_{n=1}^{\infty} 2^{-n} \min \left\{1, \sqrt{\mathbb{E}\left(\int_{\mathbb{T}_{t_{n}}} X_{u}^{2} \Delta\langle W\rangle_{u}\right)}\right\}=[X] .
\end{aligned}
$$

That is, the Brownian motion transform is a linear isometry from the space $\mathcal{L}_{0}$ of simple processes into the space $\mathcal{M}_{2}$ of right continuous, square integrable martingales. Moreover, $\mathcal{L}_{0}$ is a dense subspace of the square integrable progressively measurable processes $\mathcal{L}^{*}$ equipped with the metric $d(X, Y)=[X-Y]$, and $\mathcal{M}_{2}$ equipped with the metric $D(U, V)=\|U-V\|$ is complete. Consequently, the Brownian motion transform extends in the usual manner to an isometry from $\mathcal{L}^{*}$ into $\mathcal{M}_{2}$.

Definition 7 The stochastic dynamic integral of $X \in \mathcal{L}^{*}$ w.r.t. a Brownian motion $W$ indexed by $\mathbb{T}$ is the unique right continuous, square integrable martingale indexed by $\mathbb{T}, I(X)=\left\{I_{t}(X), \mathcal{F}_{t}: t \in \mathbb{T}\right\}$, satisfying $\left\|I\left(X^{(n)}\right)-I(X)\right\| \rightarrow 0$ for every sequence $\left\{X^{(n)}\right\}_{n=1}^{\infty}$ in $\mathcal{L}_{0}$ satisfying

$$
\left[X^{(n)}-X\right] \rightarrow 0
$$

We denote the stochastic dynamic integral of $X$ w.r.t. $W$ by $I_{t}(X)=\int_{\mathbb{T}_{t}} X_{u} \Delta W_{u}$ for all $t \in \mathbb{T}$.

Theorem 1 Let $X$ and $Y$ belong to $\mathcal{L}^{*}$, let $s, t \in \mathbb{T}$ with $t_{0} \leq s<t$, and let $\alpha, \beta \in \mathbb{R}$. Then the stochastic dynamic integral given in Definition 7 has the following properties.

1) $\int_{\mathbb{T}_{t_{0}}} X_{u} \Delta W_{u}=0$

2) $\int_{\mathbb{T}_{t}}\left(\alpha X_{u}+\beta Y_{u}\right) \Delta W_{u}=\alpha \int_{\mathbb{T}_{t}} X_{u} \Delta W_{u}+\beta \int_{\mathbb{T}_{t}} Y_{u} \Delta W_{u}$.

3) $\mathbb{E}\left(\int_{\mathbb{T}_{t}} X_{u} \Delta W_{u} \mid \mathcal{F}_{s}\right)=\int_{\mathbb{T}_{s}} X_{u} \Delta W_{u}$.

4) $\mathbb{E}\left(\left(\int_{\mathbb{T}_{t}} X_{u} \Delta W_{u}\right)^{2}\right)=\mathbb{E}\left(\int_{\mathbb{T}_{t}} X_{u}^{2} \Delta\langle W\rangle_{u}\right)=\int_{\mathbb{T}_{t}} \mathbb{E}\left(X_{u}^{2}\right) \Delta u$.

5) $\|I(X)\|=[X]$.

Proof. Properties 1, 2, and 3 follow from Definition 7. For property 4, note that

$$
\mathbb{E}\left(I_{t}^{2}(X)\right)=\mathbb{E}\left(\int_{\mathbb{T}_{t}} X_{u}^{2} \Delta\langle W\rangle_{u}\right)=\int_{\Omega} \int_{\mathbb{T}_{t}} X_{u}^{2}(\omega) \Delta\langle W\rangle_{u}(\omega) \operatorname{dP}(\omega)
$$


and from (8) we have

$$
\begin{aligned}
\int_{\Omega} \int_{\mathbb{T}_{t}} X_{u}^{2}(\omega) \Delta\langle W\rangle_{u}(\omega) \mathrm{d} \mathbb{P}(\omega) & =\int_{\Omega}\left(\int_{\mathbb{T}_{t}} X_{u}^{2}(\omega) \Delta \lambda\left(\mathbb{T}_{u}\right)\right) \mathrm{d} \mathbb{P}(\omega)+\int_{\Omega}\left(\int_{\mathbb{T}_{t}} X_{u}^{2}(\omega) \Delta\left(\sum_{b_{n} \leq u}\left(W_{b_{n}}-W_{a_{n}}\right)(\omega)\right)\right) \mathrm{d} \mathbb{P}(\omega) \\
& =\int_{\mathbb{T}_{t}} \mathbb{E}\left(X_{u}^{2}\right) \Delta \lambda\left(\mathbb{T}_{u}\right)+\sum_{b_{n} \leq t} \int_{\Omega} X_{a_{n}}^{2}(\omega)\left(W_{b_{n}}-W_{a_{n}}\right)^{2}(\omega) \mathrm{d} \mathbb{P}(\omega) \\
& =\int_{\mathbb{T}_{t}} \mathbb{E}\left(X_{u}^{2}\right) \Delta \lambda\left(\mathbb{T}_{u}\right)+\sum_{b_{n} \leq t} \mathbb{E}\left(X_{a_{n}}^{2}\right)\left(\int_{\Omega}\left(W_{b_{n}}-W_{a_{n}}\right)^{2}(\omega) \mathrm{d} \mathbb{P}(\omega)\right) \\
& =\int_{\mathbb{T}_{t}} \mathbb{E}\left(X_{u}^{2}\right) \Delta \lambda\left(\mathbb{T}_{u}\right)+\sum_{b_{n} \leq t} \mathbb{E}\left(X_{a_{n}}^{2}\right)\left(b_{n}-a_{n}\right) \\
& =\int_{\mathbb{T}_{t}} \mathbb{E}\left(X_{u}^{2}\right) \Delta\left(\lambda\left(\mathbb{T}_{u}\right)+\lambda\left(\left[t_{0}, u\right] \backslash \mathbb{T}\right)\right) \\
& =\int_{\mathbb{T}_{t}} \mathbb{E}\left(X_{u}^{2}\right) \Delta\left(\lambda\left(\left[t_{0}, u\right]\right)\right) \\
& =\int_{\mathbb{T}_{t}} \mathbb{E}\left(X_{u}^{2}\right) \Delta u
\end{aligned}
$$

Finally property 5 follows from (11) and property 4 .

Example 1 Let $\mathbb{T}=[0,1] \cup[2, \infty)$. By the definition of right-continuity of the filtration $\mathcal{F}_{t}$ given in $(7)$ note that $\mathcal{F}_{1} \neq \mathcal{F}_{2}$. Let us take a simple process

$$
X_{t}= \begin{cases}a & \text { for } t \in[0,1) \\ b & \text { for } t \in\{1\} \cup[2, \infty)\end{cases}
$$

where $a, b \in \mathbb{R}$. Then

$$
\begin{aligned}
\mathbb{E}\left(I_{2}^{2}(X)\right) & =\mathbb{E}\left(\left(a W_{1}+b\left(W_{2}-W_{1}\right)\right)^{2}\right) \\
& =a^{2}+b^{2}+2 \mathbb{E}\left(a b W_{1}\left(W_{2}-W_{1}\right)\right)
\end{aligned}
$$

and

$$
\mathbb{E}\left(\int_{\mathbb{T}_{2}} X_{u}^{2} \Delta\langle W\rangle_{u}\right)=\mathbb{E}\left(a^{2} W_{1}^{2}+b^{2}\left(W_{2}-W_{1}\right)^{2}\right)=a^{2}+b^{2} .
$$

Since by the definition of $W$ given in Section $1, W_{2}-W_{1}$ is $\mathcal{F}_{1}$ measurable and independent of $\mathcal{F}_{1}$, we observe that $\mathbb{E}\left(a b W_{1}\left(W_{2}-W_{1}\right)\right)=0$. Also

$$
\int_{\mathbb{T}_{2}} \mathbb{E}\left(X_{u}^{2}\right) \Delta u=\int_{0}^{1} a^{2} \mathrm{~d} u+b^{2} \mu(1)=a^{2}+b^{2}
$$

and hence

$$
\mathbb{E}\left(I_{2}^{2}(X)\right)=\mathbb{E}\left(\int_{\mathbb{T}_{2}} X_{u}^{2} \Delta\langle W\rangle_{u}\right)=\int_{\mathbb{T}_{2}} \mathbb{E}\left(X_{u}^{2}\right) \Delta u
$$

In the next section we present the existence and uniqueness of stochastic dynamic equation.

\section{Existence and Uniqueness}

With the theory of stochastic dynamic integral established, the second integral in (2) has meaning. Consequently, we are able to rigorously cast the IVP (1) in the integral form (2). We now consider the problem of the existence and uniqueness of solutions of (1).

Theorem 2 Let $\mathbb{T}$ be a time scale and suppose $a, b: \mathbb{T} \times \mathbb{R} \rightarrow \mathbb{R}$ satisfy the conditions

$$
\begin{aligned}
& \left|a\left(x_{1}, t\right)-a\left(x_{2}, t\right)\right| \leq L\left|x_{1}-x_{2}\right| \\
& \left|b\left(x_{1}, t\right)-b\left(x_{2}, t\right)\right| \leq L\left|x_{1}-x_{2}\right|
\end{aligned}
$$


and

$$
\begin{aligned}
& |a(x, t)| \leq L(1+|x|) \\
& |b(x, t)| \leq L(1+|x|)
\end{aligned}
$$

for all $t \in \mathbb{T}_{T}$, all $x, x_{1}, x_{2} \in \mathbb{R}$ and for some constant $L$. Let $X_{0}$ be any real-valued random variable such that $\mathbb{E}\left(\left|X_{0}\right|^{2}\right)<\infty$. Then there exists a unique strong solution $X$ of the stochastic dynamic IVP

$$
\Delta X=a(X, t) \Delta t+b(X, t) \Delta W, \quad X\left(t_{0}\right)=X_{0}
$$

on $\mathbb{T}_{T}$. Moreover, there exists a constant $k_{T} \in \mathcal{R}$ such that

$$
\mathbb{E}\left(\left|X_{t}\right|^{2}\right) \leq k_{T}\left(1+\mathbb{E}\left(\left|X_{0}\right|^{2}\right)\right) e_{k_{T}}\left(t, t_{0}\right)
$$

for $t \in \mathbb{T}_{T}$.

Proof. Let us suppose that $X$ and $\hat{X}$ are solutions of (14). Then for $t_{0}, t, T \in \mathbb{T}$,

$$
\begin{aligned}
\mathbb{E}\left(\left|X_{t}-\hat{X}_{t}\right|^{2}\right) & \leq 2 \mathbb{E}\left(\left|\int_{\mathbb{T}_{t}}\left(a\left(X_{u}, u\right)-a\left(\hat{X}_{u}, u\right)\right) \Delta u\right|^{2}\right)+2 \mathbb{E}\left(\left|\int_{\mathbb{T}_{t}}\left(b\left(X_{u}, u\right)-b\left(\hat{X}_{u}, u\right)\right) \Delta W_{u}\right|^{2}\right) \\
& \leq 2 L^{2}\left(T-T_{0}+1\right) \int_{\mathbb{T}_{t}} \mathbb{E}\left(\left|X_{u}-\hat{X}_{u}\right|^{2}\right) \Delta u \\
& \leq C \int_{\mathbb{T}_{t}} \mathbb{E}\left(\left|X_{u}-\hat{X}_{u}\right|^{2}\right) \Delta u,
\end{aligned}
$$

provided $t \in \mathbb{T}_{T}$ and $C=2 L^{2}\left(T-T_{0}+1\right)$. Therefore Lemma 2, with $C_{0}=0$, implies $\phi(t)=\mathbb{E}\left(\left|X_{t}-\hat{X}_{t}\right|^{2}\right) \equiv 0$. Thus $X_{t}=\hat{X}_{t}$ a.s. for all $t \in \mathbb{T}_{T}$.

To prove existence, let us define $X_{t}^{0}:=X_{0}$ and

$$
X_{t}^{n+1}:=X_{0}+\int_{\mathbb{T}_{t}} a\left(X_{u}^{n}, u\right) \Delta u+\int_{\mathbb{T}_{t}} b\left(X_{u}^{n}, u\right) \Delta W_{u}
$$

for $n \in \mathbb{N}_{0}$ and $t \in \mathbb{T}_{T}$. We claim that

$$
\delta_{t}^{n}:=\mathbb{E}\left(\left|X_{t}^{n+1}-X_{t}^{n}\right|^{2}\right) \leq M^{n+1} h_{n+1}\left(t, t_{0}\right)
$$

for all $n \in \mathbb{N}_{0}$, and $t \in \mathbb{T}_{T}$, where $h_{n}$ are the generalized polynomials defined by (3) and $M$ a constant that depends on $L, T$, and $X_{0}$. Indeed for $n=0$, we have

$$
\begin{aligned}
\delta_{t}^{0} & =\mathbb{E}\left(\left|X_{t}^{1}-X_{t}^{0}\right|^{2}\right) \\
& =\mathbb{E}\left(\left|\int_{\mathbb{T}_{t}} a\left(X_{0}, u\right) \Delta u+\int_{\mathbb{T}_{t}} b\left(X_{0}, u\right) \Delta W_{u}\right|^{2}\right) \\
& \leq 2 \mathbb{E}\left(\left|\int_{\mathbb{T}_{t}} L\left(1+\left|X_{0}\right|\right) \Delta u\right|^{2}\right)+2 \mathbb{E}\left(\int_{\mathbb{T}_{t}} L^{2}\left(1+\left|X_{0}\right|\right)^{2} \Delta\langle W\rangle_{u}\right) \\
& \leq M\left(t-t_{0}\right)=M h_{1}\left(t, t_{0}\right)
\end{aligned}
$$


for $M=2 L^{2}\left(T-t_{0}+1\right) \mathbb{E}\left(\left(1+\left|X_{0}\right|\right)^{2}\right)$. Under the assumption that the claim is valid for $n-1$, we have

$$
\begin{aligned}
\delta_{t}^{n} & =\mathbb{E}\left(\left|X_{t}^{n+1}-X_{t}^{n}\right|^{2}\right) \\
& =\mathbb{E}\left(\left|\int_{\mathbb{T}_{t}}\left(a\left(X_{u}^{n}, u\right)-a\left(X_{u}^{n-1}, u\right)\right) \Delta u+\int_{\mathbb{T}_{t}}\left(b\left(X_{u}^{n}, u\right)-b\left(X_{u}^{n-1}, u\right)\right) \Delta W_{u}\right|^{2}\right) \\
& \leq 2 \mathbb{E}\left(\left|\int_{\mathbb{T}_{t}}\left(a\left(X_{u}^{n}, u\right)-a\left(X_{u}^{n-1}, u\right)\right) \Delta u\right|^{2}\right)+2 \mathbb{E}\left(\left|\int_{\mathbb{T}_{t}}\left(b\left(X_{u}^{n}, u\right)-b\left(X_{u}^{n-1}, u\right)\right) \Delta W_{u}\right|^{2}\right) \\
& \leq 2\left(t-t_{0}\right) \mathbb{E}\left(\int_{\mathbb{T}_{t}}\left|a\left(X_{u}^{n}, u\right)-a\left(X_{u}^{n-1}, u\right)\right|^{2} \Delta u\right)+2 \mathbb{E}\left(\int_{\mathbb{T}_{t}}\left|b\left(X_{u}^{n}, u\right)-b\left(X_{u}^{n-1}, u\right)\right|^{2} \Delta\langle W\rangle_{u}\right) \\
& \leq 2 L^{2}\left(t-t_{0}\right) \int_{\mathbb{T}_{t}} \mathbb{E}\left(\left|X_{u}^{n}-X_{u}^{n-1}\right|^{2}\right) \Delta u+2 L^{2} \int_{\mathbb{T}_{t}} \mathbb{E}\left(\left|X_{u}^{n}-X_{u}^{n-1}\right|^{2}\right) \Delta u \\
& =2 L^{2}\left(T-t_{0}+1\right) \int_{\mathbb{T}_{t}} \mathbb{E}\left(\left|X_{u}^{n}-X_{u}^{n-1}\right|^{2}\right) \Delta u \\
& =2 L^{2}\left(T-t_{0}+1\right) \int_{\mathbb{T}_{t}} \delta_{u}^{n-1} \Delta u \\
& =2 L^{2}\left(T-t_{0}+1\right) \int_{\mathbb{T}_{t}} M^{n} h_{n}\left(u, t_{0}\right) \Delta u \\
& \leq M^{n+1} h_{n+1}\left(t, t_{0}\right),
\end{aligned}
$$

provided we choose $M \geq 2 L^{2}\left(T-t_{0}+1\right) \mathbb{E}\left(\left(1+\left|X_{0}\right|\right)^{2}\right)$ and this proves the claim. Now using (12) and(16) we have

$$
\sup _{t \in \mathbb{T}_{T}}\left|X_{t}^{n+1}-X_{t}^{n}\right|^{2} \leq 2 L^{2}\left(T-t_{0}\right) \int_{\mathbb{T}_{T}}\left|X_{u}^{n}-X_{u}^{n-1}\right|^{2} \Delta u \quad+2 \sup _{t \in \mathbb{T}_{T}}\left|\int_{\mathbb{T}_{t}}\left(b\left(X_{u}^{n}, u\right)-b\left(X_{u}^{n-1}, u\right)\right) \Delta W_{u}\right|^{2} .
$$

Consequently the martingale inequality implies

$$
\begin{aligned}
\mathbb{E}\left(\sup _{t \in \mathbb{T}_{T}}\left|X_{t}^{n+1}-X_{t}^{n}\right|^{2}\right) & \leq 2 L^{2}\left(T-t_{0}\right) \int_{\mathbb{T}_{T}} \mathbb{E}\left(\left|X_{u}^{n}-X_{u}^{n-1}\right|^{2}\right) \Delta u+8 \int_{\mathbb{T}_{T}} \mathbb{E}\left(\left|X_{u}^{n}-X_{u}^{n-1}\right|\right) \Delta u \\
& \leq C M^{n} h_{n}\left(T, t_{0}\right),
\end{aligned}
$$

by claim (17), where $C=2 L^{2}\left(T-t_{0}+4\right)$. Since

$$
\mathbb{P}\left(\sup _{t \in \mathbb{T}_{T}}\left|X_{t}^{n+1}-X_{t}^{n}\right|>\frac{1}{2^{n}}\right) \leq 4 \mathbb{E}\left(\sup _{t \in \mathbb{T}_{T}}\left|X_{t}^{n+1}-X_{t}^{n}\right|^{2}\right) \leq C 4^{n} M^{n} h_{n}\left(T, t_{0}\right)
$$

and by (4) with $4 M \in \mathcal{R}$,

$$
C \sum_{n=0}^{\infty}(4 M)^{n} h_{n}\left(T, t_{0}\right)=C e_{4 M}\left(T, t_{0}\right)<\infty
$$

the Borel-Cantelli lemma applies. Thus, $\mathbb{P}\left(\sup _{t \in \mathbb{T}_{T}}\left|X_{t}^{n+1}-X_{t}^{n}\right|>\frac{1}{2^{n}}\right.$ i.o. $)=0$. Therefore for almost every $\omega, X^{n}=$ $X^{0}+\sum_{j=0}^{n-1}\left(X^{j+1}-X^{j}\right) \rightarrow X$ on $\mathbb{T}_{T}$. Now if we let $n \rightarrow \infty$ in (16), we have

$$
X_{t}=X_{0}+\int_{\mathbb{T}_{t}} a\left(X_{u}, u\right) \Delta u+\int_{\mathbb{T}_{t}} b\left(X_{u}, u\right) \Delta W_{u} .
$$


That is (14) holds for all time $t \in \mathbb{T}_{T}$. Then we have

$$
\begin{aligned}
\mathbb{E}\left(\left|X_{t}^{n+1}\right|^{2}\right) & \leq 2 \mathbb{E}\left(\left|X_{0}\right|^{2}\right)+2 \mathbb{E}\left(\left|\int_{\mathbb{T}_{t}} a\left(X_{u}^{n}, u\right) \Delta u\right|^{2}\right)+2 \mathbb{E}\left(\left|\int_{\mathbb{T}_{t}} b\left(X_{u}^{n}, u\right) \Delta W_{u}\right|^{2}\right) \\
& \leq 2 \mathbb{E}\left(\left|X_{0}\right|^{2}\right)+2\left(t-t_{0}\right) \int_{\mathbb{T}_{t}} \mathbb{E}\left(\left|a\left(X_{u}^{n}, u\right)\right|^{2}\right) \Delta u+2 \int_{\mathbb{T}_{t}} \mathbb{E}\left(\left|b\left(X_{u}^{n}, u\right)\right|^{2}\right) \Delta u \\
& \leq 2 \mathbb{E}\left(\left|X_{0}\right|^{2}\right)+2 L^{2}\left(t-t_{0}\right) \int_{\mathbb{T}_{t}} \mathbb{E}\left(\left(1+\left|X_{u}^{n}\right|\right)^{2}\right) \Delta u+2 L^{2} \int_{\mathbb{T}_{t}} \mathbb{E}\left(\left(1+\left|X_{u}^{n}\right|\right)^{2}\right) \Delta u \\
& \leq 2 \mathbb{E}\left(\left|X_{0}\right|^{2}\right)+4 L^{2}\left(t-t_{0}\right) \int_{\mathbb{T}_{t}} \mathbb{E}\left(1+\left|X_{u}^{n}\right|^{2}\right) \Delta u+4 L^{2} \int_{\mathbb{T}_{t}} \mathbb{E}\left(1+\left|X_{u}^{n}\right|^{2}\right) \Delta u \\
& \leq 2 \mathbb{E}\left(\left|X_{0}\right|^{2}\right)+4 L^{2}\left(t-t_{0}\right)^{2}+4 L^{2}\left(t-t_{0}\right)+4 L^{2}\left(t-t_{0}+1\right) \int_{\mathbb{T}_{t}} \mathbb{E}\left(\left|X_{u}^{n}\right|^{2}\right) \Delta u \\
& \leq k_{T}\left(1+\mathbb{E}\left(\left|X_{0}\right|\right)\right)+k_{T} \int_{\mathbb{T}_{t}} \mathbb{E}\left(\left|X_{u}^{n}\right|^{2}\right) \Delta u,
\end{aligned}
$$

for some constant $k_{T} \geq \max \left\{2,4 L^{2}\left(T-t_{0}\right)\left(T-t_{0}+1\right), 4 L^{2}\left(T-t_{0}+1\right)\right\}$. Note that $k_{T}>1$. By induction, therefore,

$$
\mathbb{E}\left(\left|X_{t}^{n+1}\right|^{2}\right) \leq\left(k_{T}+k_{T}^{2} h_{1}\left(t, t_{0}\right)+\cdots+k_{T}^{n+2} h_{n+1}\left(t, t_{0}\right)\right)\left(1+\mathbb{E}\left(\left|X_{0}\right|^{2}\right)\right)
$$

and consequently by (4), we have

$$
\mathbb{E}\left(\left|X_{t}^{n+1}\right|^{2}\right) \leq k_{T}\left(1+\mathbb{E}\left(\left|X_{0}\right|^{2}\right)\right) e_{k_{T}}\left(t, t_{0}\right)
$$

for all $t \in \mathbb{T}_{T}$. If we let $n \rightarrow \infty$, then

$$
\mathbb{E}\left(\left|X_{t}\right|^{2}\right) \leq k_{T}\left(1+\mathbb{E}\left(\left|X_{0}\right|^{2}\right)\right) e_{k_{T}}\left(t, t_{0}\right) .
$$

for all $t \in \mathbb{T}_{T}$, which proves (15).

Corollary 1 Suppose that the stochastic process $X$ indexed by $\mathbb{T}$ satisfies the stochastic dynamic IVP (14) and a, b: $\mathbb{R} \times \mathbb{T} \rightarrow \mathbb{R}$ satisfy (12) and (13). Then

$$
\mathbb{E}\left(\int_{\mathbb{T}_{t}}\left|X_{u}\right|^{2} \Delta u\right)<\infty
$$

for all $t \in \mathbb{T}_{T}$.

Proof. Using (15) we have

$$
\begin{aligned}
\mathbb{E}\left(\int_{\mathbb{T}_{t}}\left|X_{u}\right|^{2} \Delta u\right) & =\int_{\mathbb{T}_{t}} \mathbb{E}\left(\left|X_{u}\right|^{2}\right) \Delta u \\
& \leq\left(1+\mathbb{E}\left(\left|X_{0}\right|^{2}\right)\right) \int_{\mathbb{T}_{t}} k_{T} e_{k_{T}}\left(u, t_{0}\right) \Delta u \\
& =\left(1+\mathbb{E}\left(\left|X_{0}\right|^{2}\right)\right)\left(e_{k_{T}}\left(t, t_{0}\right)-1\right)<\infty
\end{aligned}
$$

for all $t \in \mathbb{T}_{T}$.

Remark 1 For $\mathbb{T}=[0, \infty)$ and $t_{0}=0$, the stochastic dynamic IVP (14) reduces to the stochastic differential equation

$$
\mathrm{d} X=a(X, t) \mathrm{d} t+b(X, t) \mathrm{d} W, \quad X_{t_{0}}=X_{0},
$$

where $W$ is a Brownian motion indexed by $[0, \infty)$ and $(15)$ reduces to

$$
\mathbb{E}\left(\left|X_{t}\right|^{2}\right) \leq k_{T}\left(1+\mathbb{E}\left(\left|X_{0}\right|^{2}\right)\right) e^{k_{T} t}
$$

for all $t \in[0, T]$.

Remark 2 For $\mathbb{T}=\mathbb{N}_{0}$ and $t_{0}=0$, the stochastic dynamic IVP (14) reduces to the stochastic difference equation

$$
X_{n+1}=X_{n}+a\left(X_{n}, n\right)+b\left(X_{n}, n\right) \Phi_{n+1}, \quad X_{t_{0}}=X_{0},
$$


where $\Phi_{n+1}:=W_{n+1}-W_{n}$ are normal random variables with mean 0 and variance 1 . Likewise (15) reduces to

$$
\mathbb{E}\left(\left|X_{n}\right|^{2}\right) \leq k_{N}\left(1+\mathbb{E}\left(\left|X_{0}\right|^{2}\right)\right)\left(1+k_{N}\right)^{n-1}
$$

for all $n \in\{1,2, \ldots, N\}$.

Remark 3 For $\mathbb{T}=\left\{0=t_{0}, t_{1}, t_{2}, \ldots, t_{N}\right\}$, the stochastic dynamic IVP (14) reduces to the stochastic difference equation

$$
X_{t_{n+1}}=X_{t_{n}}+a\left(X_{t_{n}}, t_{n}\right)+b\left(X_{t_{n}}, t_{n}\right) \Phi_{t_{n+1}}, \quad X_{t_{0}}=X_{0},
$$

where $\Phi_{t_{n+1}}:=W_{t_{n+1}}-W_{t_{n}}$ are normal random variables with mean 0 and variance $\mu\left(t_{n}\right)=t_{n+1}-t_{n}$. Likewise (15) reduces to

$$
\mathbb{E}\left(\left|X_{t_{n}}\right|^{2}\right) \leq k_{t_{N}}\left(1+\mathbb{E}\left(\left|X_{0}\right|^{2}\right)\right) \prod_{n=0}^{N-1}\left(1+\mu\left(t_{n}\right) k_{t_{N}}\right)
$$

for all $n \in\{1,2, \ldots, N\}$.

Definition 8 For a function $f: \mathbb{T} \rightarrow \mathbb{R}$, let us define $\mathcal{R}_{W}$ as a space of all stochastic regressive function (w.r.t. W) such that for all $t \in \mathbb{T}$

$$
1+f(t)\left(W_{\sigma(t)}-W(t)\right) \neq 0 \quad \text { a.s. }
$$

For $\mathbb{T}=\mathbb{R}$, since $\sigma(t)=t$, we have $1 \neq 0$ which is trivially true. Likewise for $\mathbb{T}=\mathbb{Z}$, (18) reduces to $1+$ $f(t)\left(W_{t+1}-W_{t}\right) \neq 0$ a.s. for $t \in \mathbb{Z}$.

Example 2 (Stochastic exponential) Consider the stochastic dynamic IVP (14) with $a(X, t) \equiv 0$ and $b(X, t)=\beta(t) X$ for some $\beta \in \mathcal{R}_{W}$. Then according to Theorem 2, the solution of

$$
\Delta X=\beta(t) X \Delta W, \quad X\left(t_{0}\right)=X_{0},
$$

exists and is unique. For an isolated time scale $\mathbb{T}$, i.e. $\mu(t)>0$ for all $t \in \mathbb{T}$, it has been shown in (Bohner \& Sanyal, 2010) that (19) with $X_{0}=1$ has a solution $X=\mathcal{E}_{\beta}\left(\cdot, t_{0}\right)$, with the following properties:

$$
\mathbb{E}\left(\mathcal{E}_{\beta}\left(t, t_{0}\right)\right)=1 \quad \text { and } \quad \mathbb{E}\left(\mathcal{E}_{\beta}^{2}\left(t, t_{0}\right)\right)=e_{\beta^{2}}\left(t, t_{0}\right) .
$$

Now by virtue of Theorem 2 it is clear that for an arbitrary $\mathbb{T}$ the solution of (19) exists, is unique, and has properties given by (20).

Example 3 (Geometric Brownian motion) Consider the stochastic dynamic IVP (14) with $a(X, t) \equiv \alpha(t) X$ and $b(X, t)=\beta(t) X$ for some $\alpha \in \mathcal{R}$ and $\frac{\beta}{1+\mu \alpha} \in \mathcal{R}_{W}$. Then according to Theorem 2 , the solution of dynamic geometric Brownian motion

$$
\Delta X=\alpha(t) X \Delta t+\beta(t) X \Delta W, \quad X\left(t_{0}\right)=X_{0},
$$

exists and is unique. For an isolated time scale $\mathbb{T}$ it has been shown in (Bohner \& Sanyal, 2010) that (19) has a solution $X=X_{0} e_{\alpha}\left(\cdot, t_{0}\right) \mathcal{E}_{\frac{\beta}{1+\mu \alpha}}\left(\cdot, t_{0}\right)$, with the following properties:

$$
\mathbb{E}\left(X_{t}\right)=X_{0} e_{\alpha}\left(t, t_{0}\right) \quad \text { and } \quad \mathbb{E}\left(X_{t}^{2}\right)=X_{0}^{2} e_{\alpha}^{2}\left(t, t_{0}\right) e_{\frac{\beta^{2}}{(1+\mu \alpha)^{2}}}\left(t, t_{0}\right)
$$

Now using Theorem 2 it is clear that for an arbitrary $\mathbb{T}$ the solution of (21) exists, is unique, and has properties given by (22).

\section{Concluding Remarks}

In this paper, we construct the stochastic dynamic integral and the existence and uniqueness of a certain class of stochastic dynamic equation ( $\mathrm{S} \Delta \mathrm{E}$ ) on a general time scale $\mathbb{T}$. We also present examples concerning generalization of stochastic exponential and geometric Brownian motion which are used in the model of stock price behavior and to model stock prices in the celebrated Black-Scholes model. 


\section{References}

Bohner, M., \& Peterson, A. (2001). Dynamic equations on time scales. An introduction with applications. Boston, MA: Birkhäuser Boston Inc.

Bohner, M., \& Peterson, A. (Eds.) (2003). Advances in dynamic equations on time scales. Boston, MA: Birkhäuser Boston Inc.

Bohner, M., \& Sanyal, S. (2010). The stochastic dynamic exponential and geometric Brownian motion on isolated time scales, Commun. Math. Anal., 8(3), 120-135. Retrieved from http://projecteuclid.org/euclid.cma/1279647309

Grow, D., \& Sanyal, S. (2011). Brownian motion indexed by a time scale. Stoch. Anal. Appl., 29(3), 457-472. http://dx.doi.org/10.1080/07362994.2011.564441

Grow, D., \& Sanyal, S. (2012). The quadratic variation of Brownian motion on a time scale. Statist. Probab. Lett., 82(9), 1677-1680. http://dx.doi.org/10.1016/j.spl.2012.05.008

Guseinov, G. Sh. (2003). Integration on time scales. J. Math. Anal. Appl., 285(1), 107-127. http://dx.doi.org/10.1016/S0022-247X(03)00361-5

Haven, E. (2009). Quantum calculus (q-calculus) and option pricing: A brief introduction. In P. Bruza, D. Sofge, W. Lawless, K. Rijsbergen, \& M. Klusch (Eds.), Quantum Interaction, Lecture Notes in Computer Science (Volume 5494, pp. 308-314). Berlin, Heidelberg: Springer. http://dx.doi.org/10.1007/978-3-642-00834-4_26

Haven, E. (2011). Itô's lemma with quantum calculus (q-calculus): Some implications. Foundations of Physics, 41, 529-537. http://dx.doi.org/10.1007/s10701-010-9453-z

Karatzas, I., \& Shreve, S. E. (1998). Brownian motion and stochastic calculus, volume 113 of Graduate Texts in Mathematics. New York: Springer-Verlag.

Lungan, C., \& Lupulescu, V. (2012). Random dynamical systems on time scales. Electron. J. Differential Equations, 86, 1-14. Retrieved from http://www.emis.de/journals/EJDE/2012/86/lungan.pdf

Sanyal, S. (2008). Stochastic dynamic equations. (Doctoral dissertation, Missouri University of Science and Technology). Retrieved from https://mospace.umsystem.edu/xmlui/handle/10355/26429 